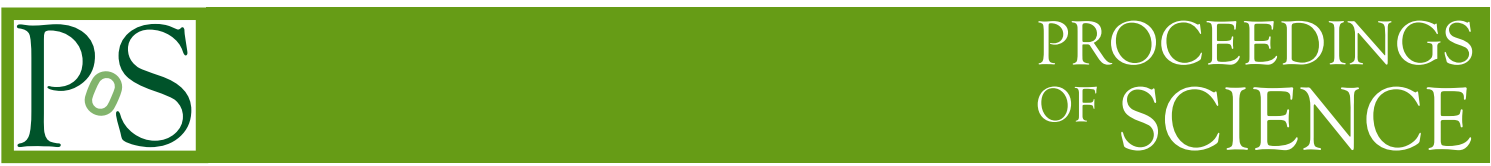

\title{
Detector considerations for a HAWC southern observatory
}

\author{
Michael DuVernois ${ }^{\dagger}$ for the HAWC Collaboration \\ $\dagger$ Department of Physics and Wisconsin IceCube Particle Astrophysics Center (WIPAC), \\ University of Wisconsin-Madison, Madison, WI, USA \\ E-mail: michael. duvernoiseicecube.wisc.edu \\ For a complete author list, see www.hawc-observatory.org/collaboration/icrc2015.php.
}

The High-Altitude Water Cherenkov (HAWC) observatory in central Mexico is currently the world's only synoptic survey instrument for gamma rays above $1 \mathrm{TeV}$. Because there is significant interest in covering the full $\mathrm{TeV}$ sky with a survey instrument, we have examined options for a Southern Hemisphere extension to HAWC. In addition to providing all-sky coverage of $\mathrm{TeV}$ sources, a southern site could complement existing surveys of the densest part of the Galactic Plane, provide continuous monitoring of Galactic and extragalactic transient sources in both Hemispheres, and simplify the analysis of spatially extended signals such as diffuse gamma rays and the $\mathrm{TeV}$ cosmic-ray anisotropy. To take advantage of the air-shower physics and lower the energy threshold of the experiment as much as possible, a high altitude site above $5000 \mathrm{~m}$ a.s.1 (vs. $4100 \mathrm{~m}$ a.s.1. at the current site in Mexico) has been specified. To facilitate efficient detector construction at such altitudes, the detector tanks would be assembled at lower altitude and delivered to the site. An all-digital communications and data acquisition scheme is proposed. Possible designs include taking advantage of digital optical module technology from the IceCube experiment as well as new custom electronics. We discuss the physics potential of such an experiment, focusing on the energy threshold, angular resolution, and background suppression capability of the experiment, as well as the advantages of full-sky coverage above $1 \mathrm{TeV}$.

Corresponding authors: M. DuVernois*

The 34th International Cosmic Ray Conference,

30 July- 6 August, 2015

The Hague, The Netherlands

\footnotetext{
${ }^{*}$ Speaker.
} 


\section{HAWC Observatory}

The HAWC Collaboration has built a high-energy gamma-ray observatory at 19 degrees North latitude and $4100 \mathrm{~m}$ a.s.l. in the Sierra Negra-Pico de Orizaba saddle valley in the State of Puebla, Mexico. The array consists of 300 water Cherenkov detector (WCD) tanks instrumented with photomultiplier tubes (PMTs) to detect and record incident gamma and cosmic ray air showers via the Cherenkov emission produced as the shower particles traverse the tank water volume. The full detector was completed and inaugurated in early 2015 with the partial detector taking data during construction.[2]

The 300 tanks and 1200 PMTs in HAWC have an active collecting area of about 12,000 $\mathrm{m}^{2}$ over a total site area of about $22,000 \mathrm{~m}^{2}$. HAWC can detect air showers with energies from about $100 \mathrm{GeV}$ to hundreds of $\mathrm{TeV}$. The direction of the primary particle is reconstructed from the arrival time distribution in the array, the energy from the PMT charge signals, and composition (distinguishing astrophysical photons from the much more abundant hadrons) from the shower topology. Charge deposited in the tanks is estimated by a pair of time-over-threshold (ToT) measurements for each PMT.

The primary physics goals of the HAWC Observatory are the detection of new TeV gamma-ray sources and the investigation of transient behaviors at $\mathrm{TeV}$. The observatory complements all-sky lower-energy coverage from the Fermi and SWIFT satellites and narrow-beam observations of the high-energy sky with Imaging Air Cherenkov Telescopes (IACT) such as MAGIC and VERITAS in the Northern Hemisphere and HESS in the Southern Hemisphere. Each day HAWC surveys about $2 / 3$ of the sky from its low latitude location.

The future of gamma-ray astronomy will be significantly different than the present with VERITAS scheduled for shutdown soon and the Cherenkov Telescope Array (CTA) collaboration looking to build IACTs in both the Northern and Southern Hemispheres. With HESS already operating and CTA soon to be building in the Southern Hemisphere, a wide field of view instrument capable of acting as a survey instrument for the enhanced deep sensitivity of CTA for the southern sky seems more than sensible. To keep pace with the evolution from VERITAS to CTA, a similar increase in effective area and sensitivity from HAWC in the north (Mexico) to HAWC in the south (HAWC-South) is required.

As an interim step for detector development, and also to enhance the ability of the HAWC array to pinpoint shower core locations when the cores are off the edge of the array, there is a plan to extend the current HAWC detector using a sparse array of "outrigger" tanks. The electronics for the outriggers will be a development platform for HAWC-South electronics efforts. The outriggers are described below and are a technological link, in both tank hardware and electronics, from HAWC to a potential larger, higher-altitude HAWC-South.

\section{Outriggers}

With the construction of the 300 HAWC tanks completed, and routine data-taking underway, the HAWC collaboration is exploring ways to increase the sensitivity of the detector. The compact array of tanks in HAWC has a large perimeter/area ratio, and this large amount of 'edge' means that a significant fraction of the high-energy showers that trigger the detector are located outside the 
main array. In the HAWC-predecessor experiment Milagro, this was mitigated with a thin sampling of small water tanks each with one PMT arrayed outside of the dense inner region. This allows for accurate core and energy measurements even for events which fall somewhat outside of the inner region of the detector. Simulations have shown that $O(100)$ small water tanks (perhaps commercial water storage tanks which are ubiquitous in Mexico) with single PMTs in each could double the effective area of the experiment.

The HAWC detector uses time-over-threshold (ToT) electronics with PMT signals transmitted to a central counting house via analog cables. The electronics were partially inherited from the Milagro experiment and new modules can no longer be produced. In addition, it is not practical to transmit analog signals over the longer distances required in an outrigger array or larger detector. Therefore, distributed electronics are the preferred solution are the preferred solution, and a simple electronics layout is shown in Fig. 1. This scheme takes advantage of inexpensive, low-power FADCs and FPGAs to digitize and then generate a total charge (integral) and time stamp (via synchronous Ethernet) which mimics the data of HAWC, but through a very different path. At HAWC, the data rate is between $25-50 \mathrm{k}$ hits/s over a dynamic range of 0.25 to $10 \mathrm{k}$ photoelectrons which constrains the availability of commercial readout schemes. The full FADC is available for calibration data, or pre-scaled event readouts, while allowing for the simpler (and HAWCcompatible) time plus charge measurement.

Fig. 2 shows the same block diagram as Fig. 1 in terms of selected, available parts. This set of electronics is undergoing prototyping, with lab versions expected operational in late 2015. The electronics are equivalent (total charge and arrival time) to the existing system at the trigger and analysis levels. However, the outrigger electronics system is more flexible, allowing for full waveforms on request, local histogramming, pre-scaling, and feature extraction. This development effort has been taking advantage of overlapping parts and requirements with the IceCube Gen2 DOM ([1]) development, for example, with common high voltage modules, shaping electronics, and similar programmable logic.

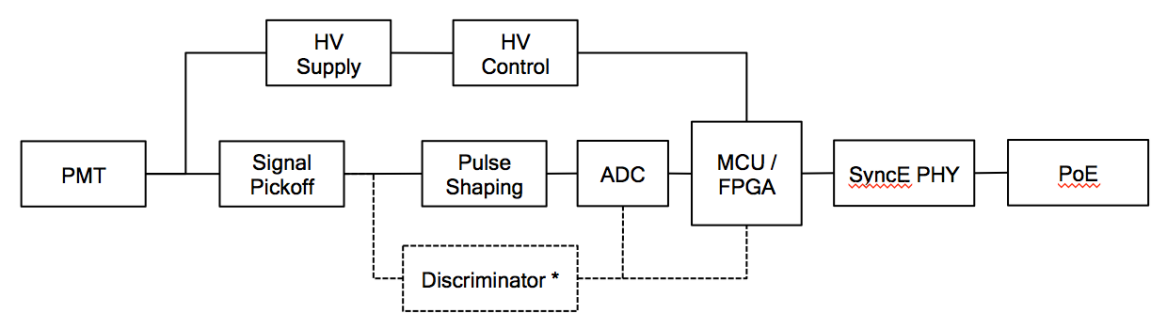

* Analog trigger optional path, otherwise trigger will be on data in the FPGA

\begin{tabular}{|c|c|c|c|c|c|}
\hline $\begin{array}{l}\text { PMT } \\
\text { Pulse }\end{array}$ & $\begin{array}{l}\text { Find Hit } \\
\text { Time }\end{array}$ & $\begin{array}{l}\text { Shape } \\
\text { Pulse }\end{array}$ & $\begin{array}{c}\text { Read } \\
\text { Waveform }\end{array}$ & $\begin{array}{c}\text { Determine } \\
\text { Charge }\end{array}$ & $\begin{array}{l}\text { Transmit } \\
\text { Data }\end{array}$ \\
\hline
\end{tabular}

Figure 1: Block diagram for the prototype outrigger electronics.

This set of electronics, and work on small commercial water tanks, could inform the design of the HAWC-South detector which, for reasons detailed below, is likely to require smaller tanks that can be transported completely outfitted (save for the water) up the mountain, and distributed lowpower (possibly solar-powered) electronics. Full waveform digitization is not ruled out however 


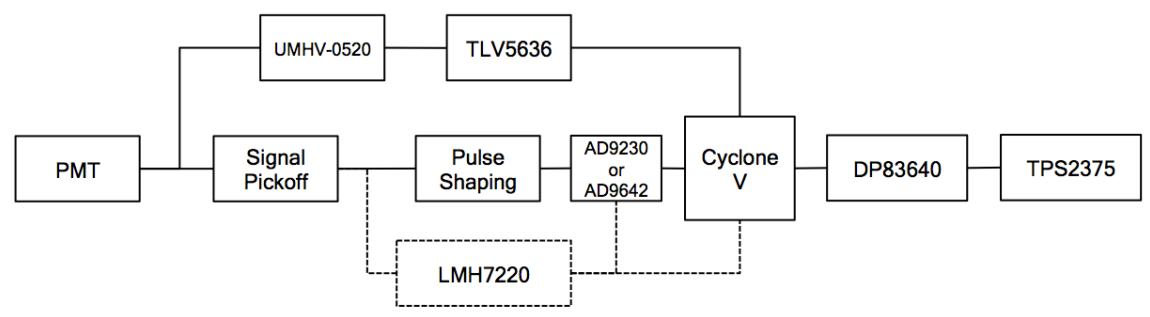

Figure 2: Chip-level hardware selections for the principle components in the outrigger electronics.

as the down-stream computing, which would be the major limiting factor right now, advances in rough accord with Moore's Law.

\section{HAWC-South}

There are four general areas of improvement in capability for a Southern Hemisphere large area water Cherenkov detector that are of interest in moving into the CTA-era of TeV gamma-ray sensitivities. These gains would be in line with the sensitivity improvements of CTA over VERITAS, or put another way, a HAWC-South would need to be large enough to act as an interesting survey instrument for CTA.

These areas of improvement are higher altitude, larger area, improved hadronic rejection, and improved shower sensitivity. Although we'll consider these one at a time, these issues are all tightly coupled to each other: for example, higher altitude inherently gives more "ground level" particles for a given primary energy, effectively improving both the shower detection sensitivity and the total effective area.

\subsection{How much higher can you get?}

Increasingly the altitude of the detector site is a huge win in terms of signal strength, lower energy threshold, and effective area, but subject to the constraints of both human physiology and land area available at a given altitude. At lower energies, the ability to cross-check with IACTs is enhanced. For a rough feel of the altitudes under consideration and the air shower profile, see Fig. 3. Sites at $5400 \mathrm{~m}$ are available in the Chilean Atacama Desert, for example the University of Tokyo Atacama Observatory is being built at $5640 \mathrm{~m}$ and a number of nearby $20,000 \mathrm{~m}^{2}$ hilltops nearby are at altitudes between 5200 and $5400 \mathrm{~m}$. The well-known cosmic-ray observatory at Chacaltaya in Bolivia is above $5200 \mathrm{~m}$ as well. (See [3] for a sensible starting point to look for existing astronomical observatories as potential Southern sites.)

At these altitudes, freezing of the detector water and the inability of unadapted personnel to work are major problems. Insulation and passive solar gain on the tanks are a potential solution to the former issue. For site-work, it seems likely that the detector (water tank, PMT, and electronics) would need to be constructed at lower altitude and then delivered to the high altitude site for later filling. Roads to the high-altitude Atacama sites appear to be reasonable; see Google Earth's imagery of the University of Tokyo Atacama Observatory or the ALMA Observatory sites. 


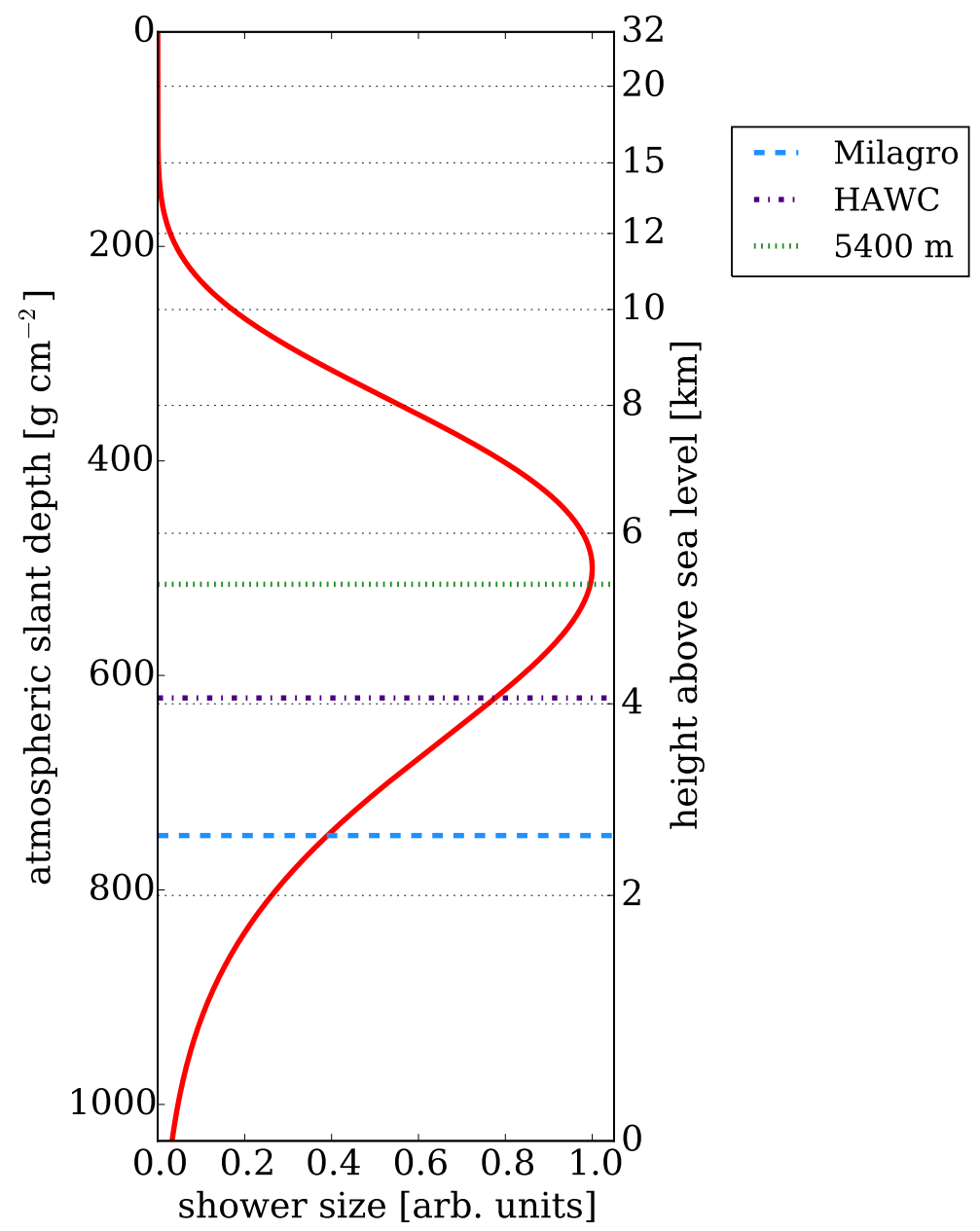

Figure 3: Air shower development with Milagro, HAWC, and potential 5400m site.

Simulations of a strawman detector were performed for a variety of altitudes $(4100 \mathrm{~m}$ for HAWC in Mexico, 5200m for plausible sites, $6000 \mathrm{~m}$ for maximum Southern altitudes of flat sites, and $6600 \mathrm{~m}$ for an extreme reference). See Fig. 4 for the ground level energy of gamma-rays below $1 \mathrm{TeV}$. The simulated detector was a $30 \times 30$ array of $1.5 \mathrm{~m}$ radius and $1.5 \mathrm{~m}$ tall tanks separated by $3 \mathrm{~m}$ and with a single 10" high-quantum efficiency PMT in the water. This detector yields estimated Crab Sensitivities as shown in Fig. 5 with the experiment sited at $20^{\circ}$ South.

Any detector at higher altitudes will have to deal with higher rates. This could be ameliorated with a larger number of smaller tanks, local coincidence requirements, or significantly faster trigger processing.

\subsection{Larger area}

A straightforward path to improved photon sensitivity is simply increasing the area of the detector array. This would need to be carefully matched to the available high altitude sites, which at above $5200 \mathrm{~m}$ seem to be constrained to less than $20,000 \mathrm{~m}^{2}$ of roughly level ground before dropping down the slopes of the hill. Likely the best route to increased area would be via lower 


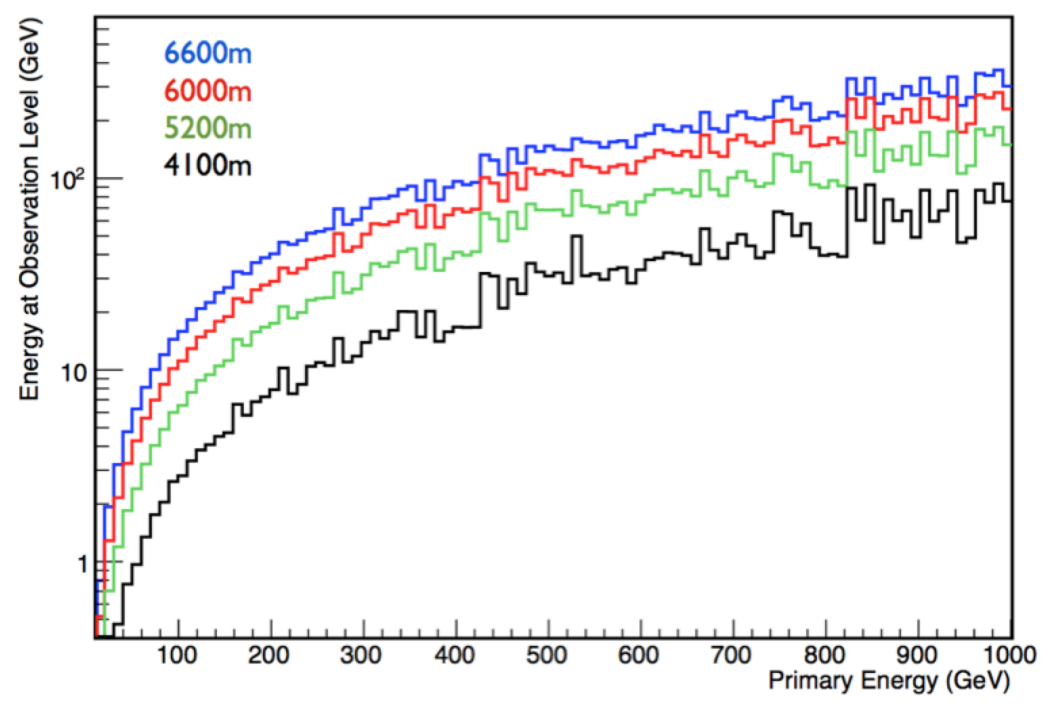

Figure 4: Surviving energy below $1 \mathrm{TeV}$.

per-tank costs of construction and instrumenting a couple of closely spaced hilltops with a common acquisition system.

\subsection{Background suppression.}

Improvements in the gamma-hadron separation directly yield improved gamma-ray rates and decreased backgrounds. With a single detector technology (the water tanks), hadron rejection comes from the shower topology. That is, hadronic cascades generate sub-shower clumps of deposited energy whereas photon showers show only the smoother electromagnetic component. Direct detection of muons and nuclear fragments in the air showers could also be used for gamma/hadron separation. Muon detection in the water Cherenkov tank, underground muon paddles, or dividing the tank into top (electromagnetic) and bottom (hadronic) segments are all options in this direction. All would effectively double the cost per tank, and the trade-off between increased numbers of tanks and increased background suppression has not yet been fully studied.

Other ideas in this direction include thin layers of liquid scintillator in thin bags within the tank, read out by a small PMT, or boron-laced plastic scintillators which observe the neutron backsplash from the Earth shortly after a hadronic shower touches down. These techniques are not yet proven in large detectors, but can also be prototyped at the HAWC Observatory in Mexico.

\subsection{Improved tank sensitivity}

Improving the sensitivity of an individual single water tank implies either increasing the light yield in the water and/or collecting more of the photons produced in the tank. The Cherenkov light yield is difficult to modify but adding in a scintillation component is possible with a mix of water and liquid scintillator (which could help prevent freezing as well). More photon collection is possible with a web of wavelength-shifting fibers dispersed through the water and light-piped to PMTs. With the high altitude of the potential sites, the amount of "field-work" at the site will need 


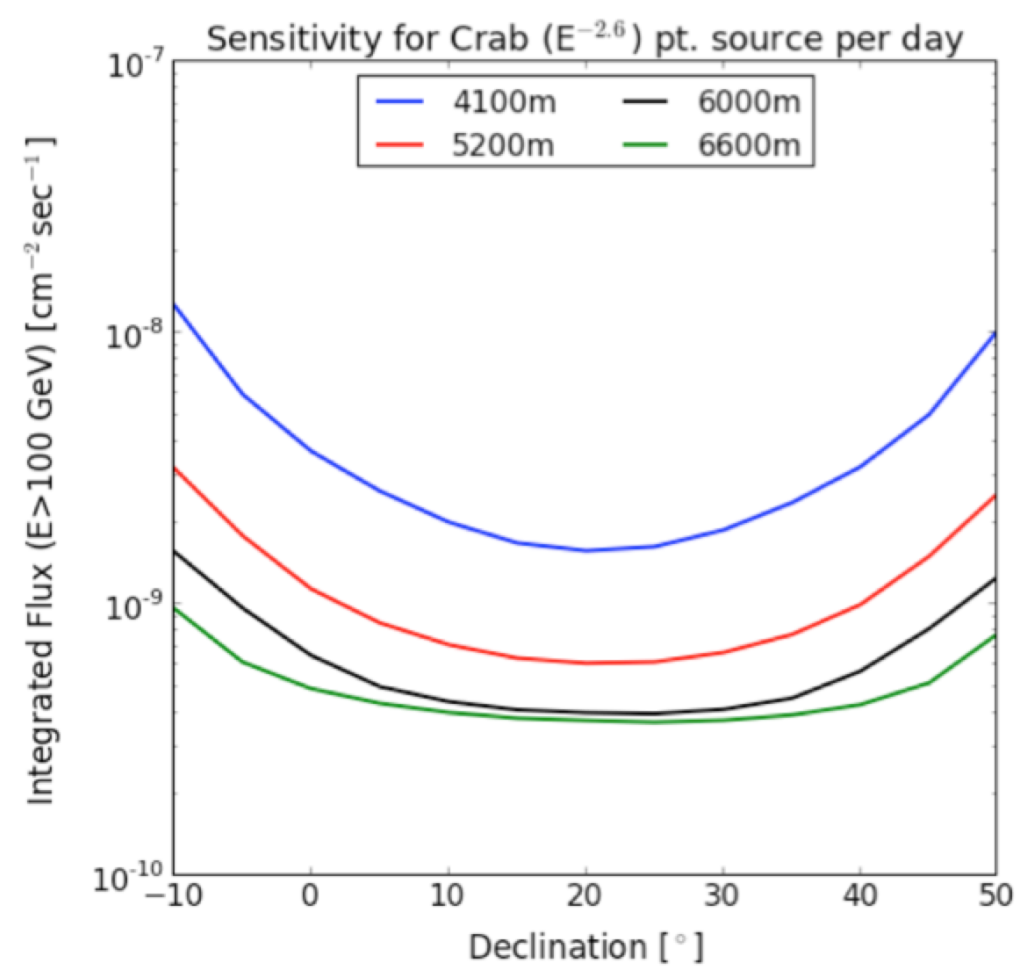

Figure 5: Sensitivity of a HAWC-like array to a gamma-ray source with the same spectrum as the Crab Nebula as a function of source declination and detector altitude.

to be kept to a minimum, so designs like this would need to be built down the mountain and taken up largely intact.

\section{Additional technologies}

In efforts to increase either the total photon yield at the PMTs, or enhancing the discrimination between the background hadrons and the signal gamma-rays, ideas such as placing scintillators above or below the tanks, adding dopants to the water, segmenting the tanks top and bottom, or adding neutron detectors (boron-doped scintillators) under the tanks are under consideration. Environmental concerns and the location of the HAWC site in Mexico, which is in a national park, have made buried detectors or dopants rather difficult to test with the existing detectors. At the HAWC site, the installation of prototype electronics and different technology detectors is relatively easy and their operation in concert with the main detector can be used for cross-calibration.

To more adequately address the testing of prototype detectors and novel detector technologies, two tanks near the counting house will be outfitted with more general readout electronics, and have time-tagging which is compatible with the HAWC time stamps but also easily available to new detectors. IceCube electronics, specially modified to handle the high data rates at the site, will be the first electronics tested in these tanks. A flexible interface standard is being designed to allow for easier access to interface with the HAWC data flow. 


\section{Schedule and prospects}

Outrigger electronics development is underway at the University of Wisconsin during 2015 with a goal of a deployable sample readout system available Spring 2016 for one of the water tanks at HAWC in Mexico. Large-scale deployments might be possible later in 2016. Parallel work taking advantage of the IceCube distributed digitization electronics is also underway with a similar prototyping time scale. Visits to potential HAWC South sites are also ongoing. With the immense potential synergies between a Southern Hemisphere large area water Cherenkov detector and the planned Southern CTA telescopes, further cooperation between the groups is foreseen.

\section{Conclusions}

The HAWC Observatory is in routine operations now, with data flowing to collaborators in the USA and Mexico and early publications are appearing (see [2] for an overview of current results). Design work is ongoing for a new set of electronics and smaller water tank setups for the outriggers intended to go around the inner core of detectors at HAWC. This design work should also help to bridge the gap between the time-over-threshold electronics located in a central counting house in HAWC (and partially inherited from the Milagro experiment) and distributed waveform digitization with local pre-analysis in a future HAWC-South design. Custom electronics are required for this due to the very high singles rate at HAWC altitudes.

There are plausible southern hemisphere sites for a detector that would be well-matched as a "finder-scope" paired to the CTA sensitivity and directionality. The sites are in South America which could work well with CTA potential sites. Alternate detector technologies to replace or augment the water Cherenkov technique are also being investigated, but would need to be sufficiently low risk to become baseline plans.

\section{References}

[1] M. A. DuVernois et al. (IceCube Gen2 Collaboration, The IceCube Gen2 Digital Optical Module and Data Acquisition System, Proc. of the 34th ICRC, The Hague, 786 (2015).

[2] J. Pretz et al. (HAWC Collaboration), Highlights from the High Altitude Water Cherenkov Observatory, Proc. of the 34th ICRC, The Hague, 866 (2015).

[3] https://en.wikipedia.org/wiki/List_of_highest_astronomical_observatories 